\title{
PROXIMAL FEMORAL PERIPROSTHETIC FRACTURE FIXATION WITH A HOOKED LOCKING PLATE: A KENYAN EXPERIENCE
}

\author{
L.N. Gakuu, MBChB, MMed (Surg), FCS (ECSA), and A. A. Admani, MBChB, MMed, Resident, Department of \\ Orthopaedic Surgery, College of Health Sciences, University of Nairobi, P. O. Box 19676 - 00202, Nairobi, Kenya \\ Correspondence to: Dr. A. A. Admani, Department of Orthopaedic Surgery, College of Health Sciences, \\ University of Nairobi, P. O. Box 19676 - 00202, Nairobi, Kenya. Email:asfadmani@yahoo.com
}

\section{ABSTRACT}

The advent and increasing use of joint replacement for various pathologies has led to a new group of fractures- the periprosthetic fractures. The hip joint is no exception to this group, a fair share of these fractures do occur around the hip joint. The management of these fractures is complex and usually needs application of basic principles to fit an individual situation rather than having a fixed set of rules. In this article we discuss the use of a hooked locking plate in the management of one such fracture.

\section{CASE REPORT}

E.M., a 70 year old lady presented to us on 25th February 2011 with complains of severe right hip pain following a fall at home. She was unable to walk and the pain was barely bearable even after taking strong analgesics. She had undergone total hip replacement in 2005 for severe osteoarthritis of the hip, but thereafter had no pain in the hip preceding this event. She also had a left foot diabetic ulcer which was infected by the time she presented to us. Her blood sugar levels was well controlled on insulin. On examination, she had a shortened right hip with external rotation. She also had a diabetic ulcer on the dorsum of the right foot.

Radiographs done showed a prosthetic right hip joint, with a spiral periprosthetic fracture of the right femur at the level of the stem, extending from the level of the lesser trochanter to just below the stem. There was no evidence of osteolysis around the stem or acetabular cup. There was no evidence of infection either. The diabetic foot was treated with the help of a chiropodist before surgical management of the fractured hip.

We finally operated on the patient on the 5th March 2011. The following was the surgical management of the patient:

(i) Exposure of the fracture site via a lateral approach. (ii) Debridement of the fracture edges.

(iii) Removal of cement from the medullary canal distally.

(iv) Trial of reduction.

(v) Placement of freshly prepared cement into the canal distally.

(vi) Absolute reduction of the fracture and cerclage wiring at the level of the spiral fracture followed by placement of a hook plate over the construct. The twin hooks of the plate were placed over the greater trochanter and the plate aligned to the lateral surface of the bone.

(vii) The fracture site was compressed using an external compression device and then locking screws were placed in all the holes in the plate. We used bicortical screws distal to the fracture site and unicortical screws at the level of the prosthesis.

(viii)The wound was closed over a drain.

Adjuvant medical and supportive therapy included:

(i) Prophylactic antibiotics- intraoperative followed by postop. for three days

(ii) Anticoagulation- both medical and non medical

(iii) Early mobilization- the patient was mobilized on the first day post operatively.

(iv) Calcium and Vitamin D supplementation 


\section{Figure 1}

Diabetic Uler of the dorsum of the right foot

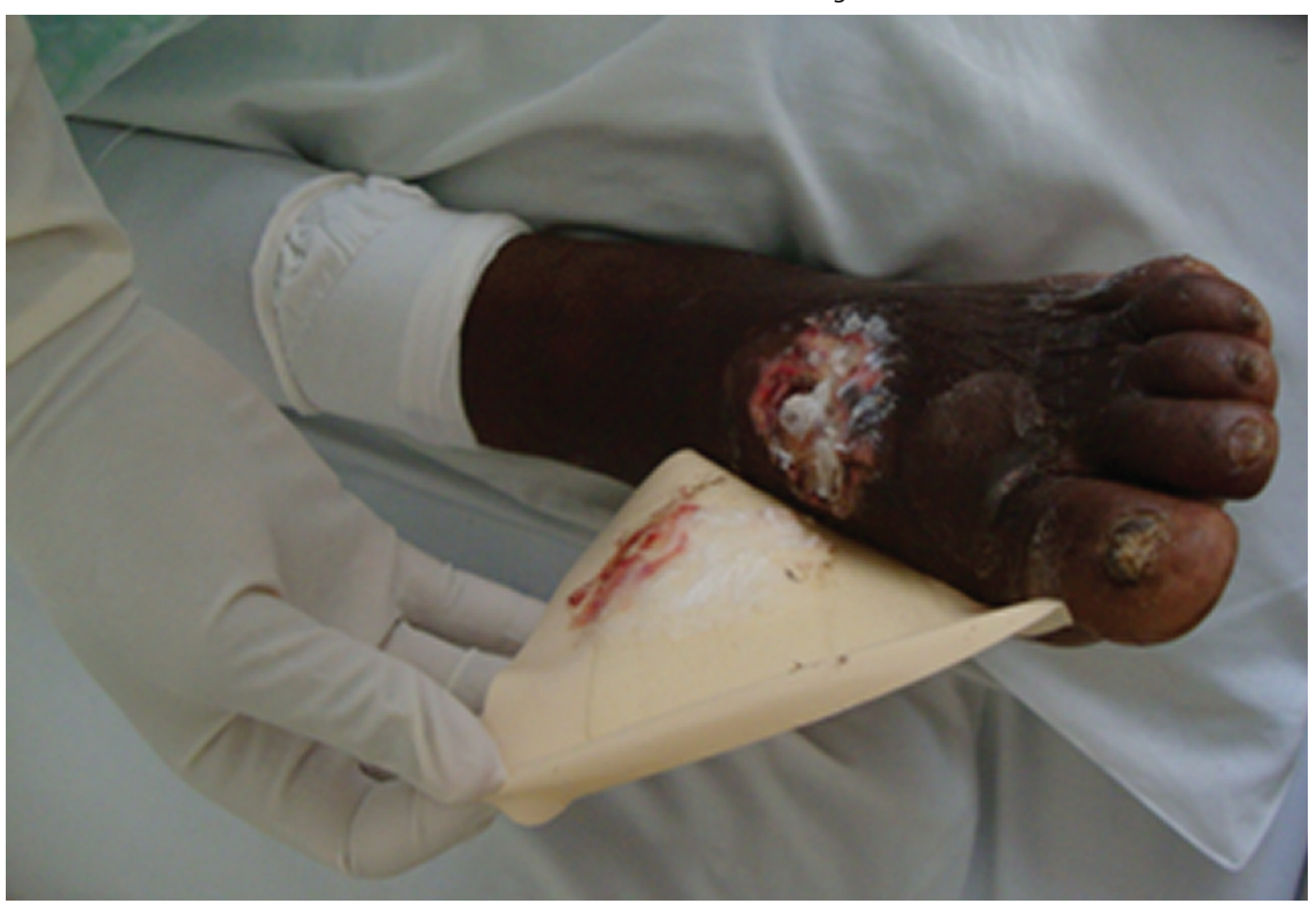

Figure 2

Close up view, showing a well fixed prosthesis and minimal osteolysis around the cement distally

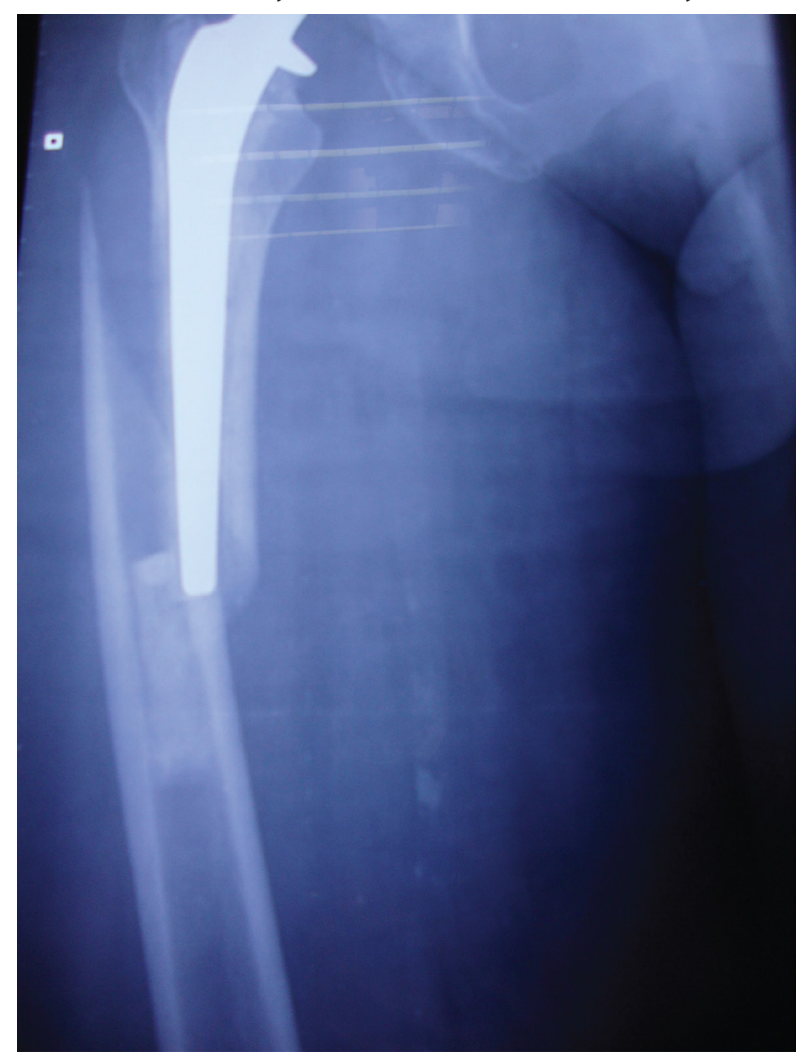

Figure 3

Pelvis radiograph showing bipolar hemiarthroplasty, and a spiral fracture at the distal end of the prosthesis

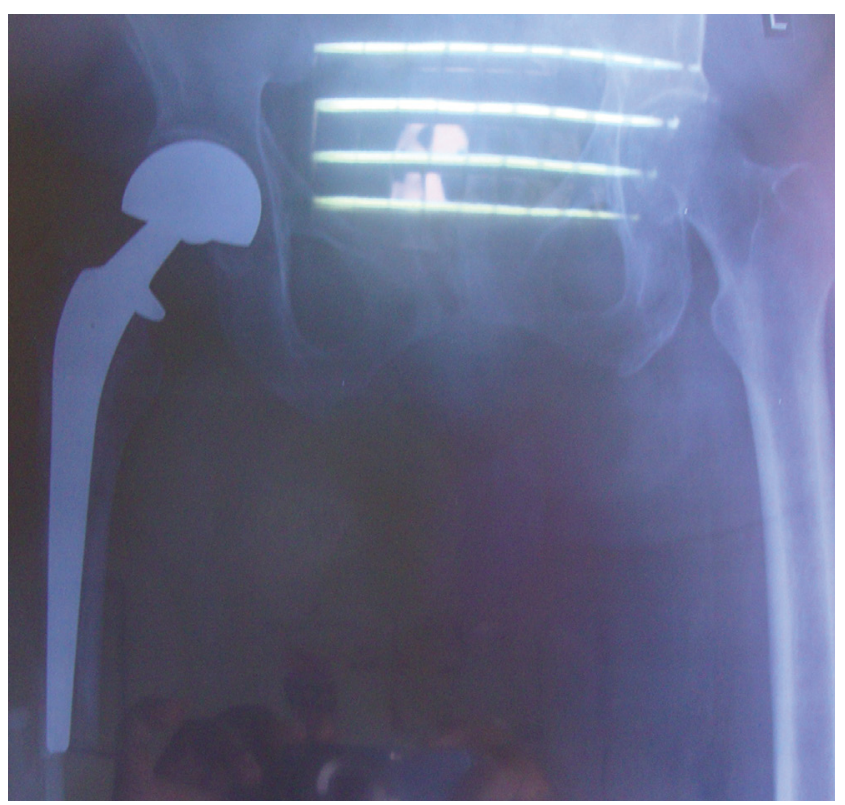

The patient was seen on 25th April 2011. she was painfree and non tender by then, and after doing a check xray, allowed partial weight bearing. 


\section{Figure 4}

Check $X$-rays confirmed excellent reduction
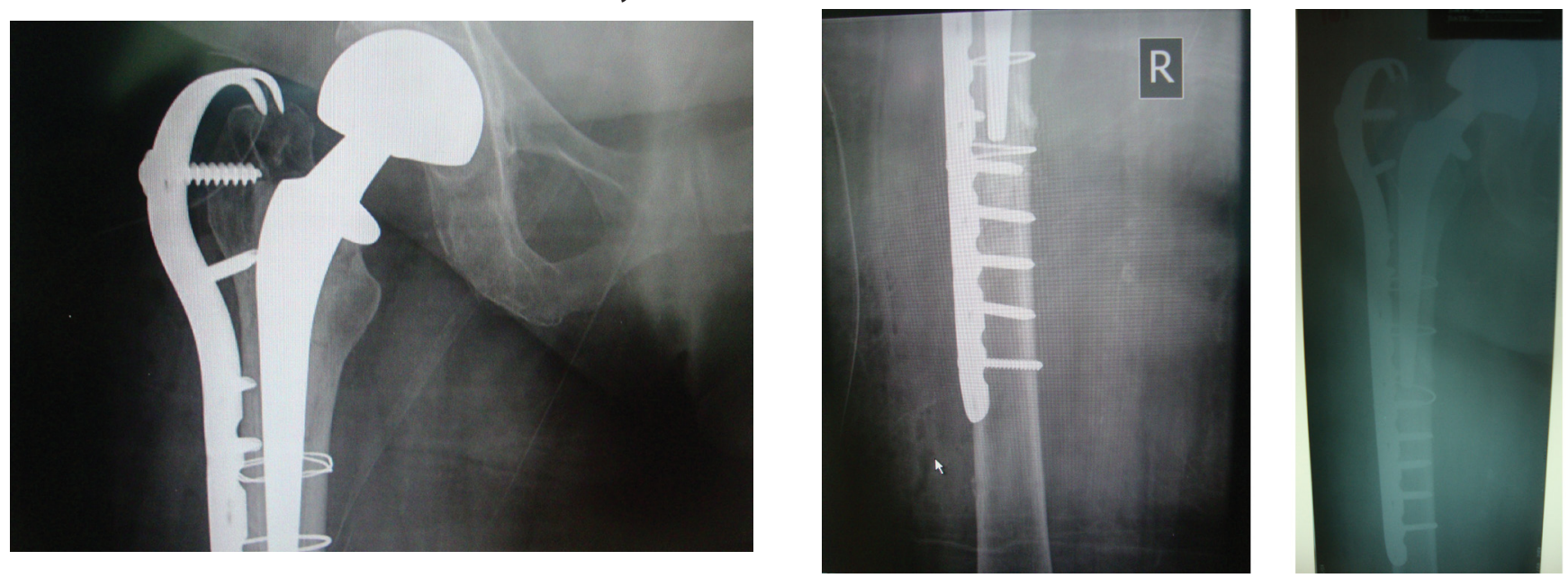

Figure 5

The following are photographs of the plate used, and some of the ancillary equipment

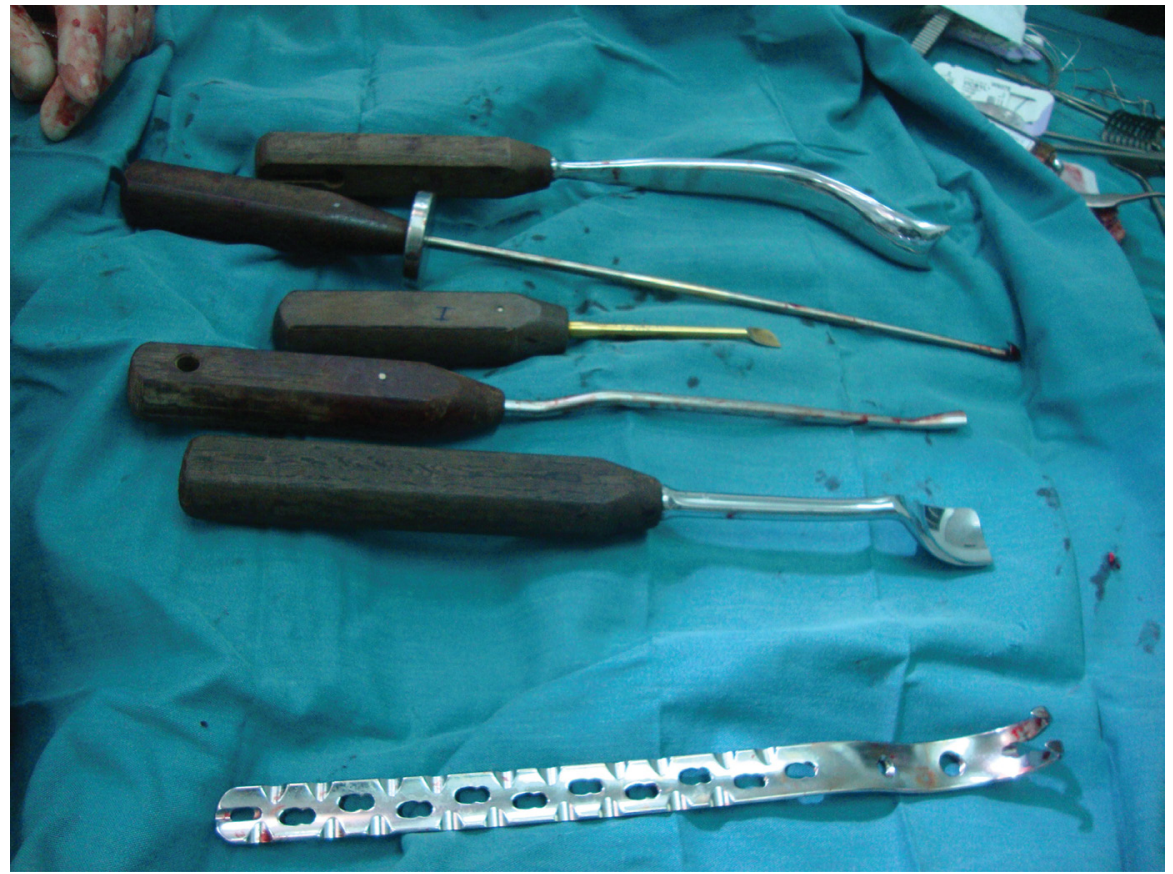

\section{DISCUSSION}

Total hip replacement arthroplasty is not a product of the twentieth century. It was first developed by Gluck in Germany in 1890. He performed a total hip replacement with ivory femoral and acetabular components cemented to bone by a combination of resin and pumice or plaster-of-Paris (1). It rose to popularity late in the twentieth century and has thereafter gained increasing popularity for a number of indications such as in inflammatory, degenerative and traumatic conditions of the hip. The increased use of hip replacement has heralded the onset of a new subset of fractures- the periprosthetic fractures(2).
Cemented total hips depend on the interface between bone-cement, and prosthesis-cement to achieve a mechanical restoration of function of the replaced part. Unfortunately this cement is not entirely inert, and is subject to wear secondary to a number of factors, mainly the hoop stresses at the bone cement interface. This wear phenomenon leads to loosening of the implant with resultant hip pain and occasionally a fracture. The prosthesis placement may also create a stress shielding effect, which weakens the bone around the region. These factors, combined with the fact that most patients undergoing hip replacement are elderly and osteoporotic leads to the perfect mix for a fracture recipe. 
Periprosthetic fractures are thus a well recognized and accepted complication of total hip replacement. The development of treatment of periprosthetic fractures around a hip replacement has come a long way from the initial conservative approach of traction, casting and bed rest. The difficulty, however, in surgical management of what would otherwise appear to be a relatively common complication of a commonly performed surgery is well illustrated by the number of classifications that have arisen to describe these fractures(3-9). Of these, the Vancouver classification (described below) is the most accepted and used. According to the Vancouver classification, periprosthetic fracture treatment decisions depend on five important factors: fracture location, stability of the implant and fracture, quality of host bone stock, patient physiology and age, and surgeon's experience(10).

Fixation may be achieved by several means. Fractures with loose stems require revision arthroplasty with a long stem prosthesis.

Fractures with a well fixed stem need fracture fixation which may be accomplished by: a lateral plate with proximal and distal cables(the Dall-Miles system(11); a lateral plate with proximal cerclage wires and distal bicortical screws(the Ogden plate(12); plating with proximal unicortical screws and distal bicortical screws; plating with proximal unicortical screws combined with cerclage and distal bicortical screws; and two allograft cortical strut grafts with cables.

In a biomechanical study by Dennis et al(13), it was found that the system of plating with distal bicortical and proximal unicortical screws with or without a cable was biomechanically the most superior of the five systems studied.

In another biomechanical study, Lever et al (14) compared the various fixation systems and found that the use of proximal unicortical and distal bicortical screws offered better stiffness and axial stability than use of cables in any instance.

The use of cement- in- cement technique has been studied in the treatment of these fractures. It is felt that this technique helps augment the fracture fixation by providing a further rigid scaffold(15).

The hook plate has been used in the treatment of subtrochanteric fractures related to periprosthetic fractures(16).

\section{The algorithm shows the Vancouver classification of postoperative periprosthetic fractures, and their preferred treatment options}

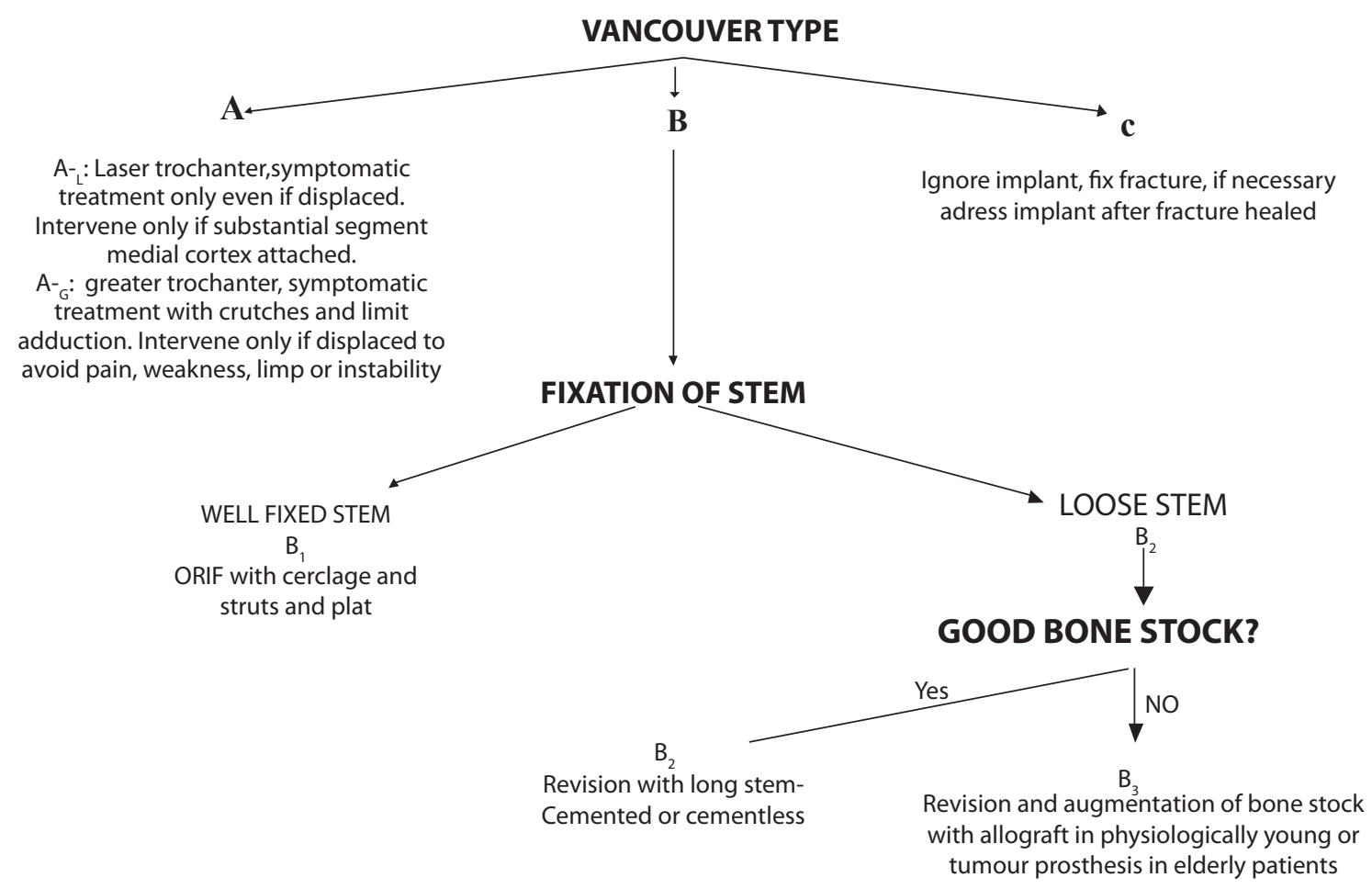


We therefore decided to use a hybridization of various techniques to achieve what we felt could be the best possible result for our patient whose fracture would fall into the B1 type by the Vancouver classification:

(i) A locking hook plate with proximal unicortical and distal bicortical screws was applied.

(ii) Cement- in cement technique was used to augment purchase of the cement.

(iii) Cerclage wires used to stabilize the fracture temporally and as part of the definitive fixation.

This fixation mode allowed early mobilization of the patient and enhanced rapid bone healing as well as to reduce the incidence of complications that plague the bedridden patient.

\section{CONCLUSION}

The fixation of periprosthetic fractures remains a challenge to even the most experienced orthopedic surgeon despite the common nature of the problem. The hybrid fixation of this particular fracture with a locked hooking plate is the first of its kind reported in Kenya. We intend to follow up on this technique with more cases and hopefully present more numbers to validate the statistical applicability of this technique.

\section{REFERENCES}

1. David, R. S. and Marvin E. S. The early history of arthroplasty in the United States. Clin. Orthop. Rel. Research 2000; 374: 55-89.

2. Ninan, T. M., Costa, M. L. and Krikler S. J. Classification of femoral periprosthetic fracture; Injury, Int. J. Care Injured 2007; 38; 661-668.

3. Beals R. K., Tower S. S. Periprosthetic fractures of the Femur; Clin. Orthop. Relat. Res. 1996; 327: 238-246.

4. Bethea, J. S., de Andrade, J. R., Fleming, L.L. et al. Proximal femoral fractures following total hip arthroplasty. Clin. Orthop. Rel. Res. 1982; 170: 95-106.
5. Brady, O. H., Kerry, R, Masri, B. A et al. The Vancouver Classification of periprosthetic fractures of the hip: a rational approach to treatment. Tech. Orthopaedi. 1999; 14(2): 107-14.

6. Cooke, P. H. and Newman, J. H. Fractures of the femur in relation to cemented hip prostheses; J. Bone Joint Surg.(Br). 1988; 70(3): 386-389.

7. Johansson, J. E, McBroom, R, Barrington T. W et al. Fracture of the ipsilateral femur in patients with total hip replacement: J. Bone Joint Surg. (Am) 1981; 63: 1435-1442.

8. Mont, M. A and Marr, D. C. Fractures of the ipsilateral femur after hip arthroplasty: a statistical analysis of outcome based on 487 patients. J. Arthroplasty 1994; 9: 511-519.

9. Whittaker, R. P., Sotos, L. N, Ralston, E. L. Fractures of the femur about endoprostheses. J. Trauma. 1974; 14: 675-694.

10. Bassam, A. M, Dominic, R. M, Clive, P. D. Periprosthetic fractures evaluation and treatment. Clin. Orthop. Rel. Res. 2004; 420: 80-95.

11. Tadross, T. S. F, Nanu, A. M, Buchanan, M. J et al. Dallmiles plating for periprosthetic $B 1$ fractures of the femur. J. Arthroplasty. 2000; 15: 48-51.

12. Huaming, Xue, Yihui, Tu, Minwei, Cai et al. Locking compression plate and cerclage band for type b1 periprosthetic femoral fractures: preliminary results at average 30-month follow-up. J. Arthroplasty (Article in Press).

13. Dennis, M. G., Jordan, A. S., Kummer F. J. et al. Fixation of periprosthetic femoral shaft fractures occurring at the tip of the stem. A biomechanical study of 5 techniques. J. Arthroplasty. 2000; 15: 523-528.

14. Lever, J. O., Zdero, $\mathrm{R}$, Nousiainen et al. The biomechanical analysis of three plating fixation systems for periprosthetic femoral fracture near the tip of a total hip arthroplasty. Mechanical Engineering Publications and Research 2010. Paper 5.

15. Richards, C. J., Duncan, C. P., Crawford RW. Cement-incement femoral revision for the treatment of highly selected Vancouver B2 periprosthetic fractures. J. Arthroplasty. 2011; 26: 335-338.

16. Cameron, H. U. Intraoperative hip fractures ruining your day. J. Arthroplasty. 2004; 19: 99-103. 